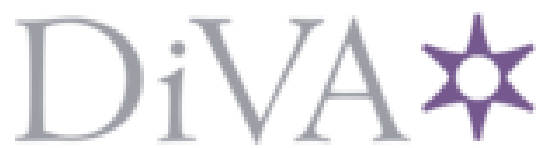

http://www.diva-portal.org

This is the published version of a paper presented at Medical Imaging 2021: Physics of Medical Imaging.

Citation for the original published paper:

Eguizabal, A., Persson, M., Grönberg, F. (2021)

A deep learning post-processing to enhance the maximum likelihood estimate of three material decomposition in photon counting spectral CT In: Proceedings of SPIE, 1159546 SPIE-Intl Soc Optical Eng https://doi.org/10.1117/12.2581044

N.B. When citing this work, cite the original published paper.

Permanent link to this version:

http://urn.kb.se/resolve?urn=urn:nbn:se:kth:diva-292428 
This is an author-posted version of the below publication. For more information see https://www.spiedigitallibrary.org/article-sharing-policies

Alma Eguizabal, Mats U. Persson, Fredrik Grönberg, "A deep learning postprocessing to enhance the maximum likelihood estimate of three material decomposition in photon counting spectral CT," Proc. SPIE 11595, Medical Imaging 2021: Physics of Medical Imaging, 1159546 (15 February 2021); https://doi.org/10.1117/12.2581044

Copyright 2020 Society of Photo-Optical Instrumentation Engineers (SPIE). One print or electronic copy may be made for personal use only. Systematic reproduction and distribution, duplication of any material in this publication for a fee or for commercial purposes, and modification of the contents of the publication are prohibited. 


\section{A deep learning post-processing to enhance the maximum likelihood estimate of three material decomposition in photon counting spectral CT}

Eguizabal, Alma, Persson, Mats, Grönberg, Fredrik

Alma Eguizabal, Mats U. Persson, Fredrik Grönberg, "A deep learning postprocessing to enhance the maximum likelihood estimate of three material decomposition in photon counting spectral CT," Proc. SPIE 11595, Medical Imaging 2021: Physics of Medical Imaging, 1159546 (15 February 2021); doi: $10.1117 / 12.2581044$

SPIE. Event: SPIE Medical Imaging, 2021, Online Only 


\title{
A deep learning post-processing to enhance the maximum likelihood estimate of three material decomposition in photon counting spectral CT
}

\author{
Alma Eguizabal $^{\mathrm{a}}$, Mats U. Persson ${ }^{\mathrm{b}}$, and Fredrik Grönberg ${ }^{\mathrm{b}}$ \\ ${ }^{a}$ Department of Mathematics, KTH Royal Institute of Technology, Stockholm, Sweden \\ ${ }^{\mathrm{b}}$ Department of Physics, KTH Royal Institute of Technology, Stockholm, Sweden
}

\begin{abstract}
Photon counting detectors in x-ray computed tomography (CT) are a major technological advancement that provides additional energy information, and improve the decomposition of the CT image into material images. This material decomposition problem is however a non-linear inverse problem that is difficult to solve, both in terms of computation expense and accuracy. The most accepted solution consists in defining an optimization problem based on a maximum likelihood (ML) estimate with Poisson statistics, which is a model-based approach very dependent on the considered forward model and the chosen optimization solver. This may make the material decomposition result noisy and slow to be computed. To incorporate data-driven enhancement to the ML estimate, we propose a deep learning post-processing technique. Our approach is based on convolutional residual blocks that mimic the updates of an iterative optimization process and consider the ML estimate as an input. Therefore, our architecture implicitly considers the physical models of the problem, and in consequence needs less training data and fewer parameters than other standard convolutional networks typically used in medical imaging. We have studied a simulation case of our deep learning post-processing, first on a set of 350 Shepp-Logan -based phantoms, and then in a 600 human numerical phantoms. Our approach has shown denoising enhancement over two different ray-wise decomposition methods: one based on a Newton's method to solve the ML estimation, and one based on a linear least-squares approximation of the ML expression. We believe this new deep learning post-processing approach is a promising technique to denoise material-decomposed sinograms in photon-counting CT.
\end{abstract}

Keywords: Photon Counting CT, material decomposition, deep learning, post-processing, maximum likelihood estimation.

\section{INTRODUCTION}

Photon-counting detectors are expected to be a great improvement for computed tomography $(\mathrm{CT})$ technology. ${ }^{1}$ One of its strong potentials is the accuracy in material discrimination in the CT image. A photon-counting detector consists of a multi-bin system with typically $B>2$ energy bins, providing enhanced X-ray spectral resolution to differentiate materials.

The material decomposition is a non-linear inverse problem typically resolved in the sinogram domain, that is, before reconstructing the projections, and using a maximum likelihood estimation ${ }^{23}$. This is a model-based technique that takes into consideration the physics model behind image formation, as well as the statistics model of the photon counts. Therefore, it is very dependent on a well-defined non-linear forward model, which is difficult to achieve. Also, the non-linearity complicates the convexity of the problem, ${ }^{4}$ and the solution, commonly iterative, may become computationally expensive. For these reasons, the material decomposition can be too slow or provide noisy results. One option to accelerate the convergence is to consider constraints in the maximum likelihood problem, although which constraints and how much these compromise the solution is a difficult issue to address. These model-based solutions to the material decomposition do not contemplate the advantages of data-driven strategies such as machine learning.

Further author information: (Send correspondence to A. Eguizabal)

A. Eguizabal: E-mail: almaeg@kth.se

Medical Imaging 2021: Physics of Medical Imaging, edited by Hilde Bosmans, Wei Zhao,

Lifeng Yu, Proceedings of SPIE Vol. 11595, 1159546 - (c) 2021 SPIE

CCC code: $1605-7422 / 21 / \$ 21 \cdot$ doi: $10.1117 / 12.2581044$

Proc. of SPIE Vol. 11595 1159546-1 
Deep learning throws in information from training data and it has been showing important success in the research of CT reconstruction algorithms ${ }^{5}{ }^{6}$ As material decomposition, Deep learning has also been proposed before the image reconstruction to perform triage and lower radiation. ${ }^{7}$ Deep learning for PCCT is an emerging research area and some authors have started using successfully neural networks in medical imaging, such as the U-net, ${ }^{8}$ to solve the material decomposition.

In this paper we propose a deep learning strategy to enhance the maximum likelihood estimate of the material decomposition in the projection domain. For this purpose, we aim for a good balance between model-based and data-driven approaches. We present a neural network post-processing strategy that mimics the structure of an iterative solution, which is the structure that leads to a good decomposition in the model-based case. Also, since it is a post-processing algorithm, we implicitly consider the physics of image generation and the statistics of the photon counts in the strategy, since the starting point is determining a maximum likelihood estimate. The computation in the sinogram domain permits to correct the decomposed materials before image reconstruction, eliminating noise and artifacts in advance, and acquiring accurate information about material concentration before applying more computation demanding reconstruction algorithms.

\section{MATERIALS AND METHODS}

In this section we first present the material decomposition problem in PCCT and how this is typically solved in a maximum likelihood estimation. Then, we describe our proposed deep learning post-processing.

\subsection{Material decomposition in Photon Counting CT}

The very good results of spectral CT provided by photon counting CT are due to the energy-resolving photon counting detectors (PCD). These detectors consists of a multi-bin system with typically $B>2$ energy bins. Each of the bins, $j=1, \ldots, B$, registers the projected energy from different sections of the energy of the X-ray spectrum, and therefore has a different energy response.

One important benefit from these energy-resolving PCD is that they allow measuring the composition of the imaged object, this is, the material decomposition. This is possible through a linear decomposition of the linear attenuation coefficient. ${ }^{9}$ Let us consider a simplified 2-dimensional $(x, y)$ image space. In order to perform a material decomposition, the X-ray linear attenuation coefficient $\mu(x, y ; E)$ is approximated by a linear decomposition into $M$ components as

$$
\mu(x, y ; E) \approx \sum_{m=1}^{M} \alpha_{m}(x, y) \tau_{m}(E),
$$

where $M$ is the number of potential materials in the image, $\tau_{m}(E)$ are the basis functions and $\alpha_{m}$ the basis coefficients. ${ }^{10}$ The material decomposition is typically performed before image reconstruction, that is, in projection domain. This permits reducing the dimensionality on the problem before reconstruction, since normally $B>M$. Also, removing the non-linearity before reconstruction and hence improving the beam-hardening of the resulting images. ${ }^{1}$ Therefore, the line integral of the basis materials

$$
a_{m}=\int_{\ell} \alpha_{m}(x, y) d s=\mathcal{R}\left(\alpha_{m}\right)
$$

are considered, where $\mathcal{R}$ is the Radon transformation operator.

Consequently, the material decomposition is a mapping from photon counts (measurement) to line integrals of basis materials ( $a$, solution). This is a non-linear inverse problem. The solution variable $a$ is a vector containing the concentrations of every material, i.e., $a=\left[a_{1}, \ldots, a_{M}\right]$. We establish the connection to the measured photon counts through a forward model of the measurement process. In this forward model the poly-chromatic BeerLambert law on each mean of counts determines the expected value of the photon counts

$$
\lambda_{j}(a)=\int_{0}^{\infty} \omega_{j}(E) \exp \left(-\sum_{m=1}^{M} a_{m} \tau_{m}(E)\right) d E .
$$


We consider a Poisson noise model in the photon counts, so each $j$ th energy component in the measured data distributes as

$$
y_{j} \sim \operatorname{Poisson}\left(\lambda_{j}(a)\right),
$$

so the measured data for every bin in contained in the vector $y=\left[y_{1}, \ldots, y_{B}\right]$.

\subsection{A Maximum Likelihood estimation}

Considering the statistical Poisson noise model, the material decomposition problem is commonly interpreted as a Maximum Likelihood (ML) estimation of the path lengths $a$. Assuming the expression of a Poisson likelihood, and after applying log-function to simplify it, the following constrained optimization problem is derived

$$
\begin{array}{cl}
\underset{a}{\operatorname{minimize}} & \sum_{j=1}^{B}\left(\lambda_{j}(a)-y_{j} \log \left(\lambda_{j}(a)\right)\right) \\
\text { subject to } & a_{i} \geq 0 \quad \forall i=1, \ldots, M
\end{array}
$$

where $a=\left[a_{1}, \ldots, a_{M}\right]$ is the vector of path lengths for materials $m=1, \ldots, M$, and the negative log-likelihood function $\mathcal{L}(a)=\sum_{j=1}^{B}\left(\lambda_{j}(a)-y_{j} \log \left(\lambda_{j}(a)\right)\right)$ is the cost function of the optimization problem. The line integrals $a$ are forced to be positive (which is true for certain basis materials) to accelerate the convergence

One way of solving the optimization problem in Eq. (5) is to consider an iterative Newton's method, where each update is calculated as

$$
a^{n+1}=a^{n}-\gamma\left[\mathrm{H} \mathcal{L}\left(a^{n}\right)\right]^{-1} \nabla \mathcal{L}\left(a^{n}\right),
$$

where $\left[\mathrm{H} \mathcal{L}\left(a^{n}\right)\right]^{-1}$ is the inverse of the Hessian matrix of the $\log$-likelihood $\mathcal{L}$. However these iterative schemes may become computational expensive. Solutions to accelerate Newton's schemes, such as an early stopping or the use of Hessian approximations, may compromise the quality of the result. We consider an interior-point method to account efficiently for the inequality constraints in the problem. ${ }^{11}$

\subsection{Linearized approximations of the ML}

Even though there are optimization tools to accelerate the convergence to solve (5), iterative solutions may become too time-consuming. One way of increasing the speed considerably is using a linearized approximation of the forward model, as proposed by Alvarez. ${ }^{12}$ The counterpart of a fast linear solution is that the quality of the results may not be good enough.

In this paper we have considered a simplified version of the solution in ${ }^{12}$ (that is, we do not consider the lookup-table step nor the weighted cost). This much faster alternative considers a linear approximation, assuming a first order Taylor series expansion to approximate the non-linear forward model and Gaussian noise. In such a case, the optimization problem simplifies into a linear least-squares expression, which is computationally inexpensive but may be too simplistic and result in bias and suboptimal noise performance. We also consider this solution in our analysis, with a closed-form solution similar to $a_{\text {linear }}=\left(T T^{*}\right)^{-1} T^{*} z$, where $T$ is a matrix calculated from the Taylor expansion of the forward model as

$$
T_{j m}=\frac{\int_{0}^{\infty} \tau_{m} \omega_{j}(E) \exp \left(-\sum_{i=1}^{M} a_{i} \tau_{i}(E)\right) d E}{\int_{0}^{\infty} \omega_{j}(E) \exp \left(-\sum_{i=1}^{M} a_{i} \tau_{i}(E)\right) d E}
$$

and $z$ a log-function of the photon counts (we refer to ${ }^{12}$ for more details of this approximation).

\subsection{A deep learning post-processing}

Deep learning and available training data can improve the solutions to the material decomposition. A data-driven post-processing can regularize and denoise the output of the optimization methods in a very time-efficient way. Furthermore, to compensate for the small training databases in medical imaging, we aim for a deep learning architecture that takes into consideration the structure of the problem, and balances the model-based and the data-driven. 
Therefore, in order to enhance the solution with deep learning, we propose a set of convolutional residual blocks. Each block consists in a set of convolutional layers followed by rectified linear unit activations, as depicted in Fig. 1. We interpret these learned blocks as a few more iterative steps of an optimization solver: the update function (which is a function of the Hessian matrix in a Newton's scheme, for instance, as in Eq. (6)) is replaced by a learned neural network $\Psi$, that is

$$
a^{n+1}=a^{n}-\Psi_{\theta^{n}}\left(a^{n}\right),
$$

where $\Psi_{\theta^{n}}\left(a^{n}\right)$ is a function representing the operation of the $n$th residual block, parametrized by $\theta^{n}$ (the values of the convolution filters that are learning in the training). We illustrate the network architecture in Fig. 1.

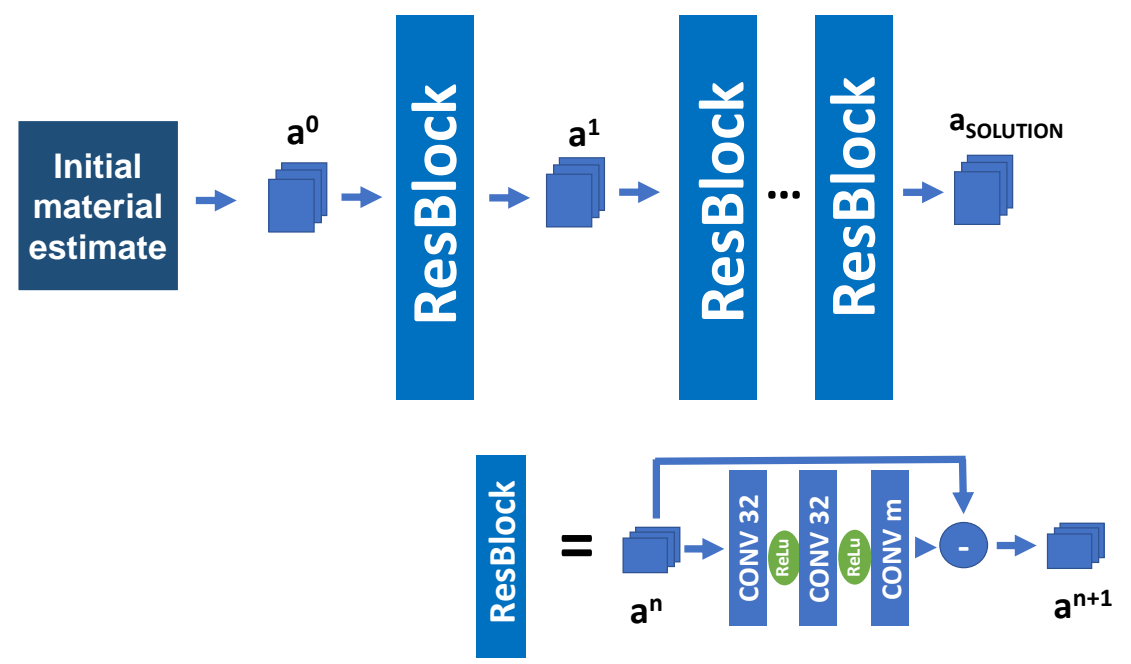

Figure 1. Proposed post-processing network architecture, that consists of a set of convolutional layers in residual blocks (ResBlocks) that mimic the updates of an iterative approach.

This post-processing technique is not specific for a particular initial material decomposition solution. In our study we implement it for the previously described Newton-based ML estimation (good, but compromised by acceleration) and for the linearized approximation (very fast to compute but low quality).

\section{RESULTS AND DISCUSSION}

The proposed deep learning requires a training database. In order to validate our proposed post-processing, we have performed two simulation test: a proof-of-concept with small Shepp-Logan phantoms, and a more realistic scenario with antropomorphic numerical phantoms. We also apply the post-processing to both described solutions to the material decomposition:

1. The ML estimate with an interior-point method (as described in section 2.2): an iterative model-based solution to the material decomposition that is still noisy.

2. The linearized approximation (as described in section 2.3): a one-step solution that is very fast to compute, but the quality is not acceptable.

\subsection{A simulation study}

In order to simulate a PCD, we first consider a model for the combined response of the detector (an 8-bin detector, i.e., $B=8)$ and a $120 \mathrm{kVp}$ source. ${ }^{13}$ We assume a three material decomposition $(M=3)$ with bone (calcium), soft-tissue (water) and iodine as contrast agent. For the attenuation responses of the materials we use data from NIST database.

We also simulate the material images separately for each material. We create a series of phantoms (as described in the next sections). Then, these are transformed to the projection domain (material sinograms). 
For that, we have assumed a fan-beam geometry, and defined the forward model of the imaging system as an operator and a Radon transformation, every step using the ODL library. ${ }^{14}$ We choose a filtered back projection (FBP) to calculate the inverse Radon transformation in our analysis.

With respect to the deep learning scheme, we consider our proposed post-processing architecture in section 2.4. We define the neural network in Pytorch with 5 ResBlocks $(N=5$ learned iterations) for the Shepp-Logan experiment and 10 ResBlocks ( $N=10$ learned iterations) for the human phantom experiment. We choose Adam optimizer and train the networks on one NVIDIA GPU GeForce RTX 2080 Ti for a sufficient number of epochs.
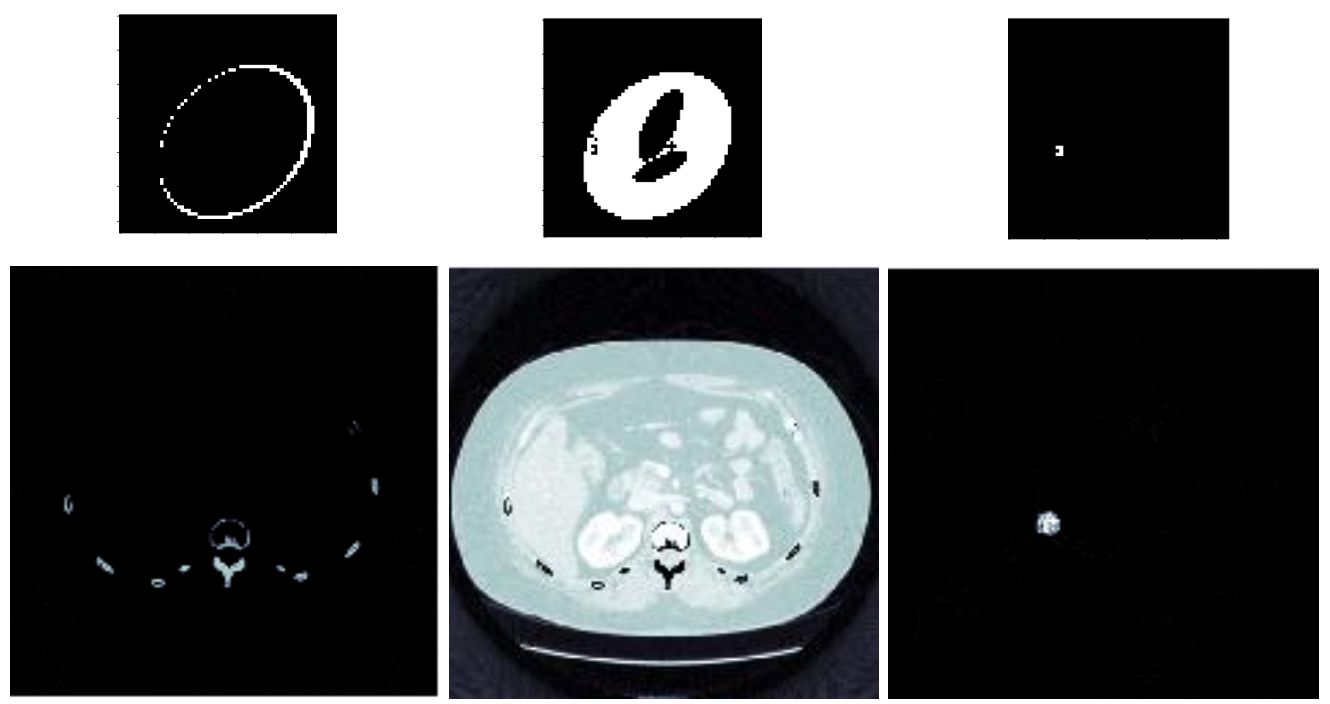

Figure 2. One example our the simulated material images from the Shepp-Logan dataset (first row) and the human phantom database (second row). Three material images are simulated: bone (first column), soft-tissue (second) and iodine (third).

\subsubsection{Shepp-Logan experiment}

Our first experiment consists in a proof-of-concept with a simple phantom: the Shepp-Logan head. In order to generate the training and test datasets, we consider the three materials with uniform concentrations, and assume the external ellipsoids are bone, the brain tissue is soft-tissue and the tumours are iodine regions. To add variability to the data, we have randomly changed the size, rotation and iodine regions position. One example of this phantom dataset is presented in Fig. 2.

We have simulated a set of 350 Shepp-Logan phantoms of 64 x 64 pixels in image space and $100 \times 100$ pixels in projection space. We consider 250 samples to train and 100 to test.

\subsubsection{Antropomorphic numerical phantom experiment}

Our next step to validate our technique is to consider more realistic antropomorphic numerical phantoms (human phantoms). To this purpose we have used the KiTS19 Challenge kidney dataset. ${ }^{15}$ In these images, the abdominal area of the patient, containing the kidneys is present. To simulate the material images we establish a threshold to diferentiate the bone and the rest of tissue, based on the Hounsfield units of the CT volumes in KiTS19. The kidneys are already segmented in the database, as well as the tumoral region masks. Therefore, the three materials considered: bone and soft-tissue after thresholding, and iodine as contrast agent in the masked tumours. The simulated values of $a$ are concentration-based. For bone and soft-tissue these are approximated directly from the Hounsfield units. For the iodine, the concentration is placed on the tumor regions with a random distribution, between 0 and $10 \mathrm{mg} / \mathrm{ml}$. One example of the human phantoms is shown in Fig. 2.

We simulate 600 of theese human phantoms. The image size is $35 \times 35 \mathrm{~cm}$ in $512 \times 512$ pixels and a radiation dose of approximately $100 \mathrm{mAs}$. A flat approximation of an $9 \mathrm{~mm}$ Aluminum filter is also simulated. We consider 
512 angles and 512 detector elements in the projection domain. From these samples, 500 are used as training samples and 100 as test.

\subsection{Analysis of results}
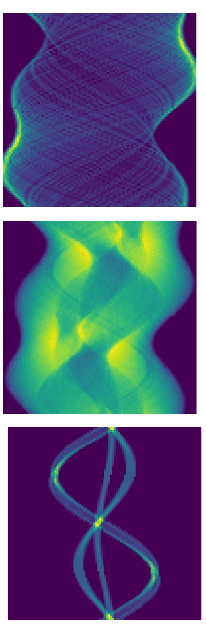

(a)
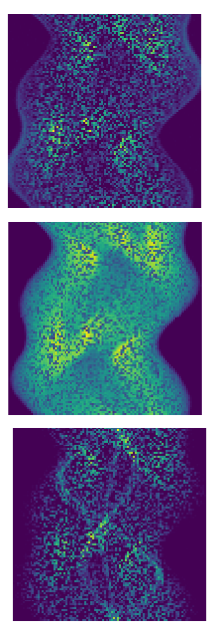

(b)
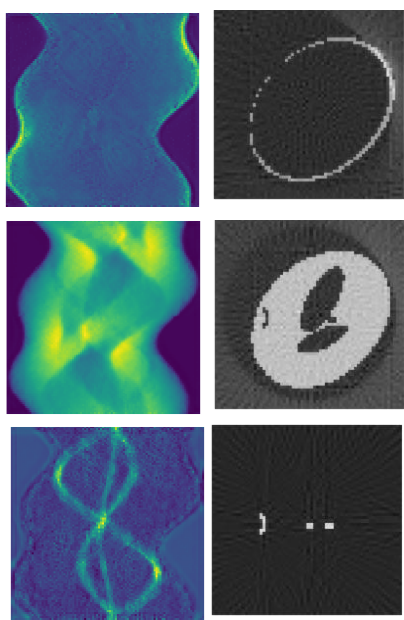

(c)

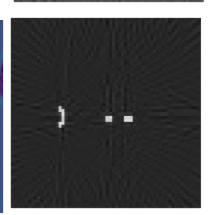

(d)
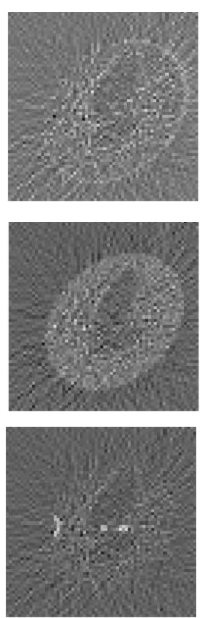

(e)
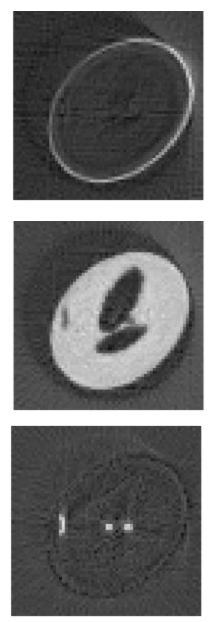

(f)

Figure 3. Shepp-Logan phantom experiment: qualitative results of post-processing the interior point -based ML estimate. (a) True sinograms of materials for bone (first row), soft-tissue (second row) and iodine (third row). (b) Solution of an interior point method. (c) Proposed denoised sinograms. (d) True basis images after reconstruction with filtered back-projection (FBP). (e) interior point-based solution after FBP. (f) Proposed denoised sinograms after FBP.

\begin{tabular}{|l||l|l|l|l|}
\hline \hline Shepp-Logan & Interior point & Interior point + DL & Linear & Linear + DL \\
\hline$\Delta$ PSNR & - & $+20 \mathrm{~dB}$ & - & $+30 \mathrm{~dB}$ \\
MSE & 0.5755 & 0.0066 & 6.5095 & 0.0179 \\
error mean & 0.0738 & 0.0009 & 0.4086 & 0.0275 \\
\hline
\end{tabular}

Table 1. Shepp-Logan experiment: quantitative results. We evaluate both the interior point-based ML solution (Interior point) and the linear approx.-based ML solution (Linear). We present the increase of PSNR (peak-to-noise ratio) after deep learning (+ DL) denoising, as well as the MSE (mean squared error) and error mean (bias).

\begin{tabular}{|l||l|l|l|l|}
\hline \hline Human & Interior point & Interior point + DL & Linear & Linear + DL \\
\hline$\Delta$ PSNR & - & $+15 \mathrm{~dB}$ & - & $+22 \mathrm{~dB}$ \\
MSE & 0.7802 & 0.0334 & 129.7641 & 1.2848 \\
error mean & 0.0299 & 0.0026 & 2.9963 & 0.2359 \\
\hline
\end{tabular}

Table 2. Human phantom experiment: quantitative results. We evaluate both the interior point-based ML solution (Interior point) and the linear approx.-based ML solution (Linear). We present the increase of PSNR (peak-to-noise ratio) after deep learning ( + DL) denoising, as well as the MSE (mean squared error) and error mean (bias).

Our proposed data-driven post-processing technique provides very good results in both the Shepp-Logan and the human phantom tests. The quality of the ML solutions (both the iterative and the linearized) are considerably improved after our deep learning approach.

Our initial study with the Shepp-Logan phantoms showed very promising results. In Table 1 we present the quantitative results over the 100 test samples, and how our deep learning post-processing enhances both an interior point ML estimate and a linear approximation-based ML one. We present the increase in PSNR (peak-to-noise ratio), the MSE (mean-squared error) and the error mean (bias). We also show one example of a test sample in Fig. 3 for the post-processing of the interior point-based ML. 

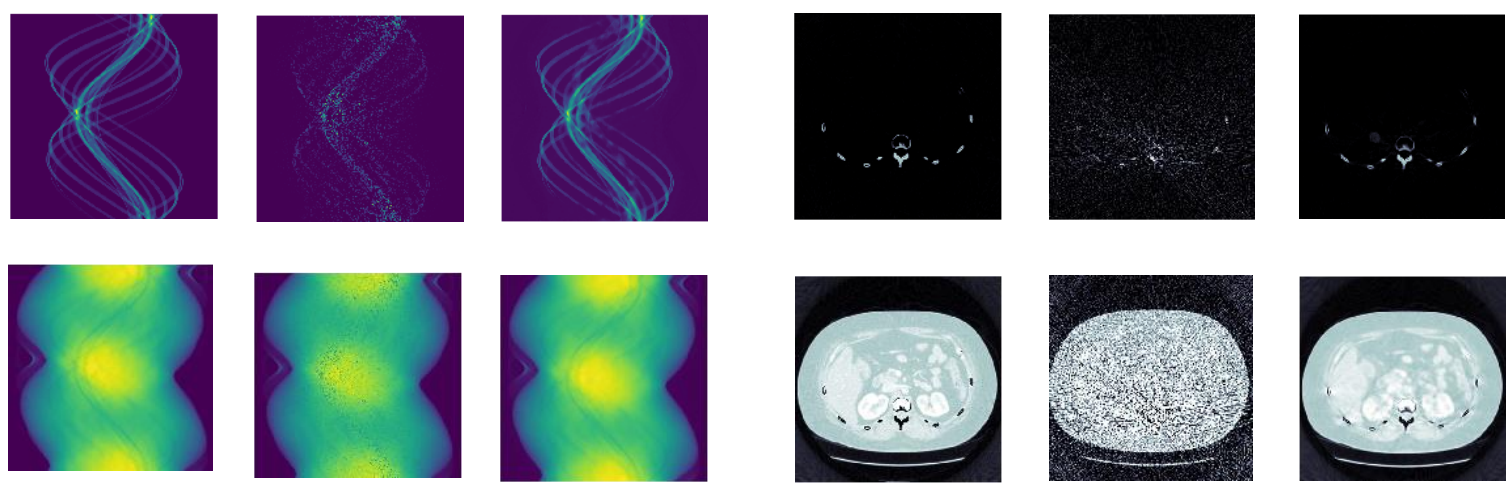

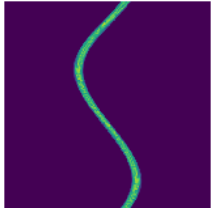

(a)

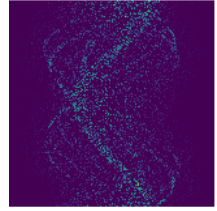

(b)

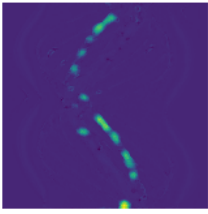

(c)

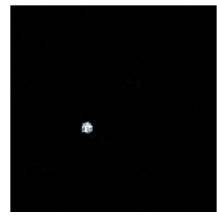

(d)

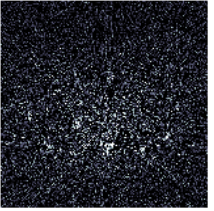

(e)

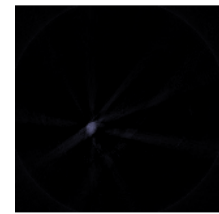

(f)

Figure 4. Human phantom experiment: qualitative results of post-processing the interior point -based ML estimate. (a) True sinograms of materials for bone (first row), soft-tissue(second row) and iodine (third row). (b) Solution of an interior point method. (c) Proposed denoised sinograms. (d) True basis images after reconstruction with filtered back-projection (FBP). (e) interior point-based solution after FBP. (f) Proposed denoised sinograms after FBP.

The results are also very encouraging in the human phantom study. A very good performance is shown with this more realistic phantoms, even though the texture, shapes and material concentrations are more challenging. The PSNR, MSE and error mean are presented in table 2. We see that a $+15 \mathrm{~dB}$ improvement in PSNR is achieved, and the MSE and bias are one order of magnitude smaller after the post-processing in the case on the interior point ML (first two columns). The post-processing also provides a very significant improvement to the bad quality of the linearized ML solution (+30 dBs of PSNR, and significantly reduced MSE and error mean). One example of a test sample with an interior point ML estimate, before and after the post-processing, is depicted in Fig. 4. The linearized ML post-processing (Fig. 5) is also presented for a qualitative evaluation of the technique.

The interior-point ML estimate together with the deep learning post-processing presents better results than the combination of Linear ML and deep learning. We believe that this first choice is a better trade-off between computation time and ML estimation quality. The linearized ML post-processed results could be better: from visually inspecting Fig. 5 we see how the iodine detection seems worse than the other two materials. Anyway, the improvement provided by the neural network scheme is impressive given the bad quality of this linearized ML solution. This approximation is probably too simplistic for this test case: it was designed for two materials only, and it requires a calibration correction and a weighting in the least-squares cost that we have ignored. We still believe that a low quality (and fast to compute) ML estimate could be enhanced with our deep learning post-processing and reach a very good accuracy with very little computational cost.

In order to visualize the impact of the post-processing improvement in a clinical use, we calculate a virtual mono-energy image using the material decomposed sinograms after an FBP reconstruction. On these, we overlay the concentrations of bone (blue) and iodine (red). We present five samples of our test dataset in Fig. 6, with different patient sizes and positions of the tumor. We observe the significance of our deep-learning post-processing to select a tumor with iodine in the reconstructions. Before the post-processing, the noise and cross-talk from other materials in the iodine sinogram produces an "explosive" and noisy mapping of the idoine, which makes the 

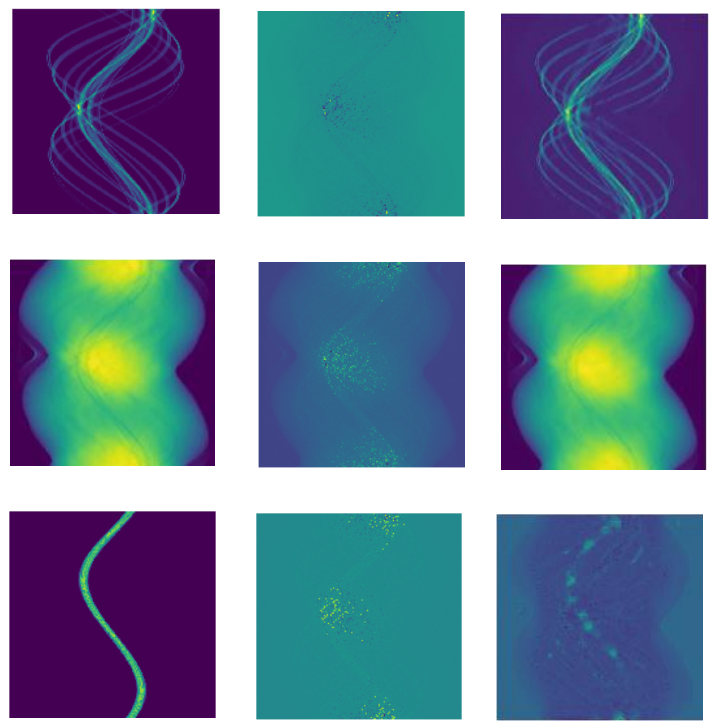

(a)

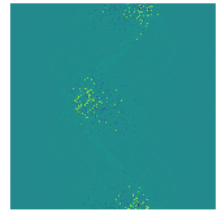

(b)

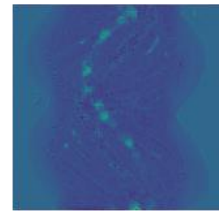

(c)
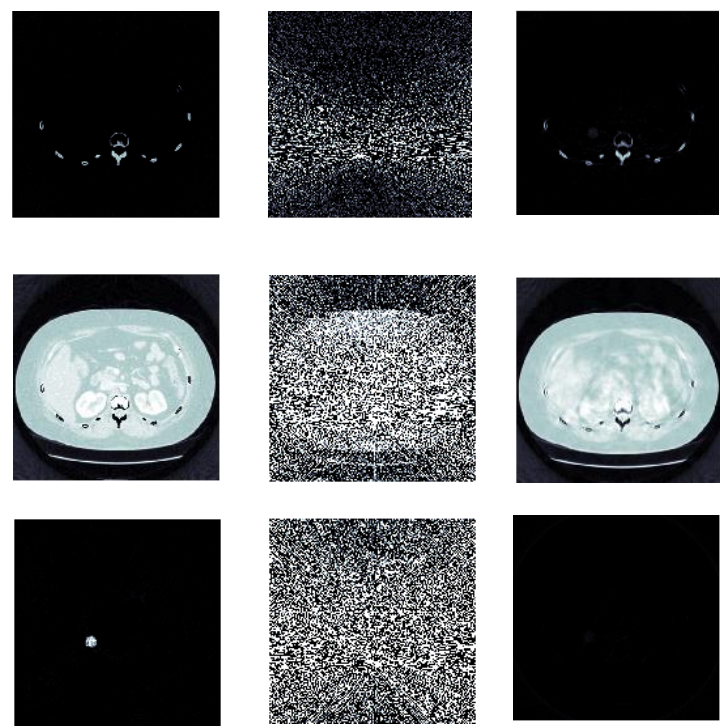

(d)
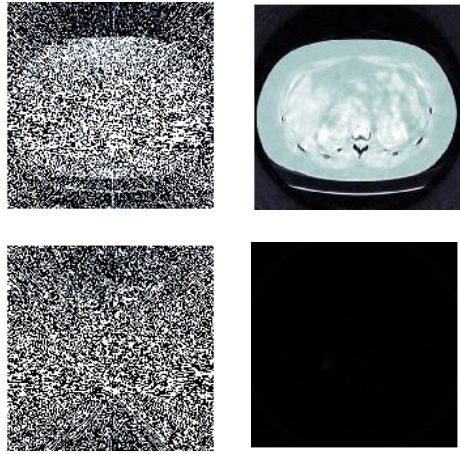

(e)

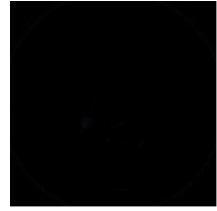

(f)

Figure 5. Human phantom experiment: qualitative results of post-processing the linearly approximated ML estimate. (a) True sinograms of materials for bone (first row), soft-tissue (second row) and iodine (third row). (b) Solution of a linearized approximation of the ML estimation. (c) Proposed denoised sinograms. (d) True basis images after reconstruction with filtered back-projection (FBP). (e) linearised solution after FBP. (f) Proposed denoised sinograms after FBP.

clinical evaluation very complicated. Our post-processing techniques corrects for these artifacts, and enhances significantly the clinical interpretation of the results.

Furthermore, this proposed neural network does not have as many parameters to learn as other typically used in medical imaging (around $10^{5}$, which is orders of magnitude smaller than U-Net, with $10^{7}$ parameters, or VGG16, with more than $10^{8}$ ). Then, with a relatively small training set (250 in the Shepp-logan case, and 500 with the human phantom) we obtain the presented very good results.

\section{CONCLUSIONS}

We have proposed a deep learning post-processing algorithm to improve the maximum likelihood estimate of the material decomposition in projection domain. Deep learning is a promising tool that can provide data-driven enhancement to model-based solutions to the material decomposition in photon counting CT.

As neural network architecture, we have designed a sequence of residual convolution blocks to mimic an iterative solution to an optimization problem. The previous ML estimation implicitly provides information about the physics and statistical models to the architecture. The network also takes into account the iterative scheme that leads to a good solution, in contrast to more commonly used architectures in medical imaging.

We have conducted a simulation study to validate our deep learning strategy obtaining very good results. We have improved considerable the initial ML estimate of the material decomposition, not only with Shepp-Logan phantoms and homogeneous concentrations, but also with human phantoms containing complex and realistic textures and concentrations.

This proposed neural network is a simple and easy-to-train framework, that does not require big amounts of training data to show considerable denoising power (250 training samples in this proof-of-concept and 500 in the human phantom simulation), and it does not demand significant additional computational time. Furthermore, the fact that the deep learning denoising is also able to correct the linear-approximation based method holds 
(a)
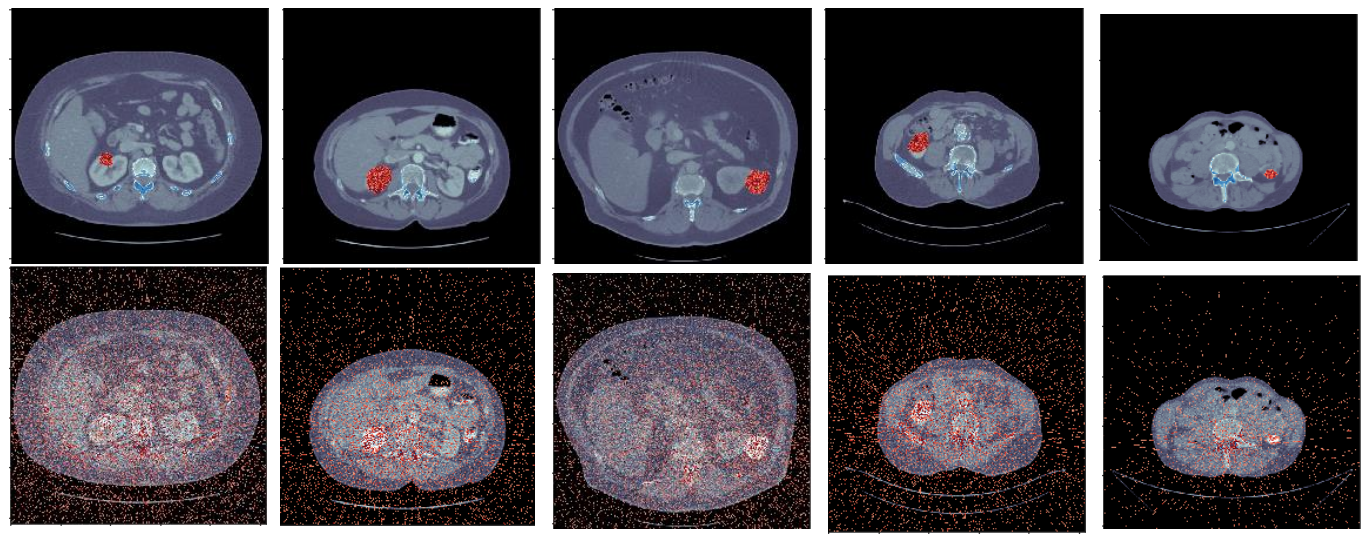

(b)
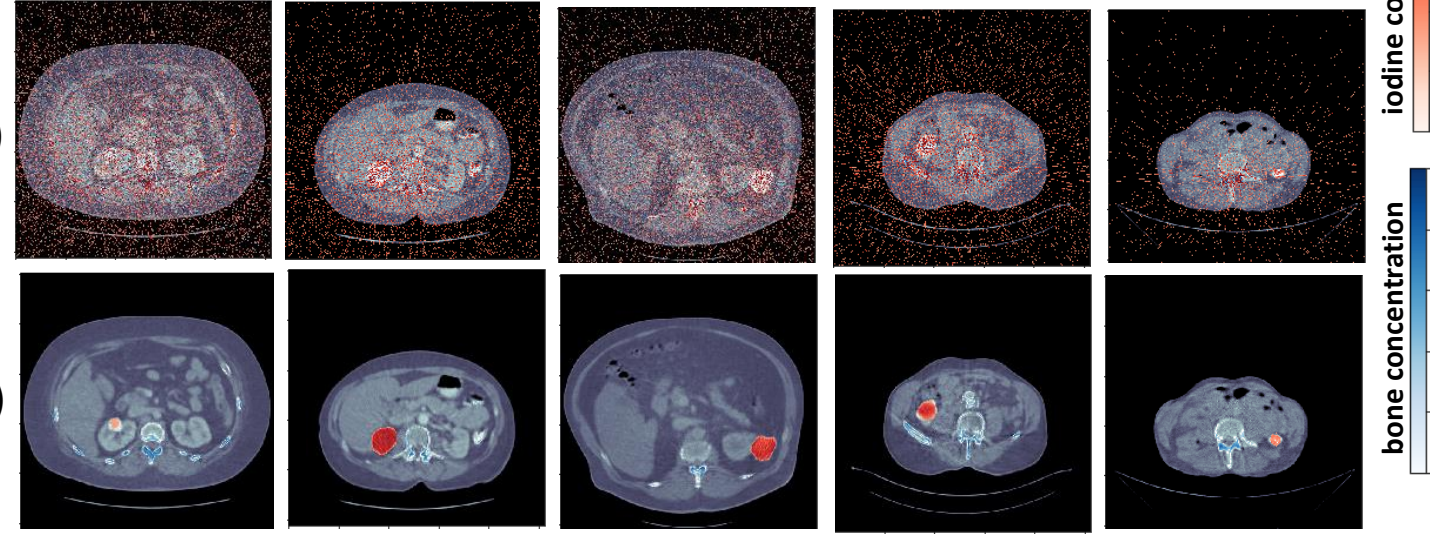

Figure 6. Virtual mono-image with material concentration overlay calculated from (a) The ground-truth material singrams + FBP, (b) The interior point -based ML material estimates + FBP, (c) The previous one post-processed with our proposed deep learning strategy.

promise for a very fast decomposition technique that could potentially be robust to model errors and a noisy decomposition. Nevertheless, a good ML estimation has provided better results after the post-processing.

In a future study we will evaluate whether the deep learning network may also learn the mismatching errors between the forward model used in the ML estimate and the actual true and unknown forward operator of the measuring system.

\section{ACKNOWLEDGMENTS}

This work has received funding from the Swedish Foundation of Strategic Research under Grant AM13-0049, from MedtechLabs and from the European Union's Horizon 2020 research and innovation programme under the Marie Sklodowska-Curie grant agreement No 795747. The authors disclose past financial interests in Prismatic Sensors AB and research collaboration with GE Healthcare. The authors would also like to thank Ozan Öktem and Mats Danielsson for providing insightful discussions and nice ideas.

\section{REFERENCES}

[1] Danielsson, M., Persson, M., and Sjölin, M., "Photon-counting x-ray detectors for ct," Physics in Medicine Biology (2020).

[2] Si-Mohamed, S., Daniel Bar-Ness, M. S., Tatard-Leitman, V., Cormode, D. P., Naha, P. C., Coulon, P., Rascle, L., Roessl, E., Rokni, M., Altman, A., Yagil, Y., Boussel, L., and Douek, P., "Multicolour imaging with spectral photon-counting ct: a phantom study," European Radiology Experimental 2, 34 (2018).

[3] Grönberg, F., Lundberg, J., Sjölin, M., Persson, M., Bujila, R., Bornefalk, H., Almqvist, H., Holmin, S., and Danielsson, M., "Feasibility of unconstrained three-material decomposition: imaging an excised human heart using a prototype silicon photon-counting ct detector," European Radiology 30, 5904-5912 (2020).

[4] Abascal, J. F. P. J., Ducros, N., and Peyrin, F., "Nonlinear material decomposition using a regularized iterative scheme based on the bregman distance," Inverse Problems 34, 124003 (oct 2018).

[5] Adler, J. and Öktem, O., "Learned primal-dual reconstruction," IEEE Transactions on Medical Imaging 37(6), 1322-1332 (2018). 
[6] Wang, G., Ye, J. C., Mueller, K., and Fessler, J. A., "Image reconstruction is a new frontier of machine learning," IEEE Transactions on Medical Imaging 37(6), 1289-1296 (2018).

[7] Lee, H., Huang, C., Yune, S., Tajmir, S. H., Kim, M., and Do, S., "Machine friendly machine learning: Interpretation of computed tomography without image reconstruction," Scientific Reports 9(15540) (2019).

[8] Abascal, J., Ducros, N., Pronina, V., Bussod, S., Hauptmann, A., Arridge, S., Douek, P., and Peyrin, F., "Material decomposition problem in spectral ct: a transfer deep learning approach," in [2020 IEEE 17th International Symposium on Biomedical Imaging Workshops], (Apr. 2020).

[9] Roessl, E. and Proksa, R., "K-edge imaging in x-ray computed tomography using multi-bin photon counting detectors," Physics in Medicine and Biology 52, 4679-4696 (jul 2007).

[10] Alvarez, R. E. and Macovski, A., "Energy-selective reconstructions in x-ray computerized tomography," Physics in medicine and biology 21(5), 733-744 (1976).

[11] Boyd, S. and Vandenberghe, L., [Convex Optimization], Cambridge University Press (2004).

[12] Alvarez, R., "Estimator for photon counting energy selective x-ray imaging with multibin pulse height analysis," Medical Physics 38(5), 2324-34 (2011).

[13] Persson, M., Wang, A., and Pelc, N. J., "Detective quantum efficiency of photon-counting CdTe and Si detectors for computed tomography: a simulation study," Journal of Medical Imaging 7(4), 1 - 28 (2020).

[14] Adler, J., Kohr, H., and Öktem, O., "Operator discretization library (odl)," (Jan. 2017).

[15] Heller, N., Isensee, F., Maier-Hein, K. H., Hou, X., Xie, C., Li, F., Nan, Y., Mu, G., Lin, Z., Han, M., et al., "The state of the art in kidney and kidney tumor segmentation in contrast-enhanced ct imaging: Results of the kits19 challenge," Medical Image Analysis, 101821 (2020). 